\title{
Vibration Reduction of Wind Loads using a Tuned Liquid Damper
}

\author{
Sun-Young Paek \\ Department of Architecture Engineering \\ Chonbuk National University \\ Jeonju, Korea \\ Byung-Hee Nam \\ Department of Architecture Engineering \\ Chonbuk National University \\ Jeonju, Korea
}

\author{
Ki-Pyo You* \\ Department of Architecture Engineering, Long-Span \\ steel Frame system Research Center \\ Chonbuk National University \\ Jeonju, Korea \\ e-mail: youkp@jbnu.ac.kr \\ * Corresponding Author \\ Young-Moon Kim \\ Department of Architecture Engineering, Long-Span \\ steel Frame system Research Center \\ Chonbuk National University \\ Jeonju, Korea
}

\begin{abstract}
Extensive study has taken place concerning the reduction of wind-induced vibrations in tall buildings. One method to mitigate this effect adds an auxiliary damping device to the building to increasing the damping ratio of the primary structure, that reducing wind-induced vibration. In the present study, passive tuned liquid damper(TLD) installed on top of an aeroelastic building model which had a different side ratio of 2 and an aspect ratio of 8 , was tested in a wind tunnel. The boundary layer flow representing a suburban area was simulated. Wind direction to the small face of the building model was normal, and the root-mean square (RMS) across-wind displacements at the top of the building model, caused mainly by motion-induced vortex, were measured and showed a reduction in the effect of motion-induced vortex excitations of tall building. Nine TLDs, with have a mass ratio of 1-2\% and frequency ratio of 0.95-1.05 were used for the experiment. The test results showed that maximum TLD effects for the reduction of vortex-induced excitations occur at a mass ratio of $2 \%$ and a frequency ratio of $1 \%$.
\end{abstract}

Keywords-Wind load; Vibration table; High-rise; Excitation direction

\section{INTRODUCTION}

Many modern tall buildings have been constructed using high-strength materials with lower damping ratios. Those tall buildings may be prone to vortex-induced excitation at wind speeds below the design wind velocity value. Although the structural system of tall buildings is designed to satisfy the strength-safety requirements, the excitation might cause discomfort to the building occupants.

Therefore, many researchers and engineers have investigated methods for mitigating such wind-induced excitations and improve the performance of tall buildings against wind loads. Most of their efforts concentrated on aerodynamic modification of building shape and structural modifications using auxiliary damping devices, such as passive, active, and semi-active damping to reduce windinduced excitations $[1,2,3]$. One of them is installing tuned liquid damper(TLD) at the top floor level of the building without exterior power controlling it. Such a TLD system is recognized as more effective and economic than that of tuned mass damper(TMD). The experimental research introducing TLDs to civil structures was carried out by Modi et al. [4] in the 1980s. Noji et al. [5] investigated the characteristics of rectangular types of TLD with deep water in which a net is installed to achieve a suitable damping ratio. Fujino et al. [6] studied the characteristics of rectangular and circular types of TLDs using a shaketable test. In their paper, to investigate the effect of TLD reducing root-mean square (RMS) across-wind displacement responses, including motion-induced vortex excitations of tall buildings to wind loads, wind tunnel tests using aeroelastic building models (side ratios of 2 and 3) with and without TLD was carried out. RMS acrosswind displacement responses of the building model with TLD were reduced to $30 \%$ of those of the building model without TLD.

\section{WINDTUNNEL TEST}

The length of the wind tunnel test equipment was $12 \mathrm{~m}$, its width $1.2 \mathrm{~m}$, and its height $1.5 \mathrm{~m}$, while the range of variable wind speed was $0.5 \sim 20 \mathrm{~m} / \mathrm{s}$. The boundary layer flow representing natural wind over suburban terrain was simulated. The power law exponent coefficient of the mean longitudinal wind speed profile is 0.15 , and the longitudinal turbulence intensity is about $10 \%$ at the top of the building model. Fig. 1 shows the vertical distributions of the mean longitudinal wind velocities and the longitudinal turbulence intensities. The present study examined the characteristics of vibration responses due to wind speed changes by means of a gimbal system that can analogize the dynamic characteristics (m, c, k) of a highrise building. The block ratios of the measuring section within the wind tunnel for the aeroelastic model were within the range of $5 \%$ : they were $3.16 \%$ (the side ratio 2 ) Fig. 2 represents the vibration responses due to damping ratio and wind speed changes of the high-rise building. The results of the test demonstrated that vortex-induced vibrations occurred when wind speed and damping ratio of the high-rise building was low. However, the vortex- 
induced vibrations are supposed to disappear when the damping ratio of a high-rise building increases. In general, the damping ratio of a high-rise building is within the range of $0.5 \sim 3 \%$ in accordance with the characteristics of used materials. In order to control vortex-induced vibrations occurring in the low wind speed section of a high-rise building with a low damping ratio, this study carried out an aeroelastic experiment based on wind speed changes after installing a TLD water tank on top of the model. In addition, it examined the relation of the optimal damping ratio with the mass and natural frequency of the TLD installed in the high-rise building. Tab .1 represents the results of the simulation between the original building and the aerodynamic vibration model. Tab .2 shows the specification of the aeroelastic vibration model for the TLD experiment. The natural frequency of the TLD is determined by the height of the water in the water tank. A total of nine types of TLDs installed in the aeroelastic vibration model were used for the test in accordance with the vibration frequency ratios $(0.95,1$, and 1.05$)$ and the mass ratios (1, 1.5 and 2$)$. The frequency ratio means the frequency of the TLD to the natural frequency of the aeroelastic vibration model. The mass ratio refers to the mass of the TLD to the generalized mass of the aeroelastic vibration TLD model. Table 3 displays the size, frequency ratio, and mass ratio of the TLD tank used for the test. This study examined changes in the natural frequency of the experimental model after the TLD was installed on top of the aeroelastic vibration model. A free vibration experiment was carried out for the aeroelastic vibration model.

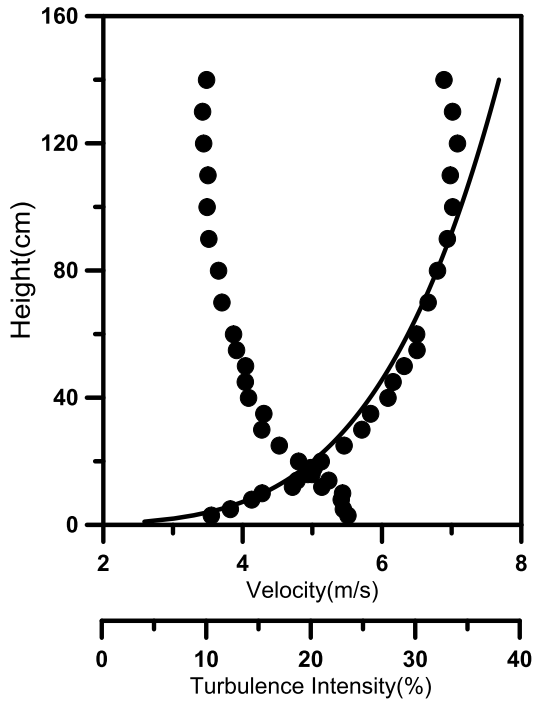

Figure 1. Vertical distribution of the mean longitudinal velocities and the longitudinal turbulence intensities

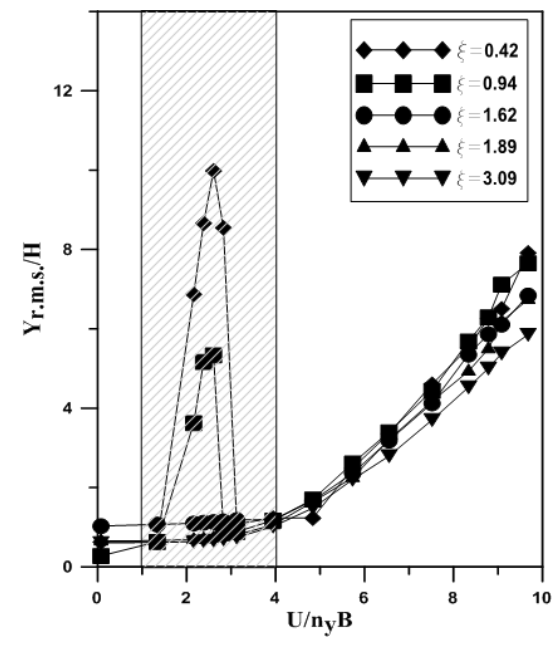

Figure 2. Response of across-wind direction

TABLE I. LAW OF SIMULATE BETWEEN PROTOTYPE AND BASIC MODEL OF THE STRUCTURE

\begin{tabular}{|c|c|c|c|}
\hline & Prototype & Model & Scale \\
\hline Height $(\mathbf{m})$ & 160 & 0.8 & $1 / 200$ \\
\hline Cross section $\left(\mathbf{m}^{2}\right)$ & 403.7 & 0.0100 & - \\
\hline Natural Frequency $(\mathbf{H z})$ & 0.3 & 3 & 10 \\
\hline Building density $\left(\mathbf{k g} / \mathbf{m}^{3}\right)$ & 120 & 120 & 1 \\
\hline
\end{tabular}

TABLE II. PROPERTIES OF AEROELASTIC MODEL

\begin{tabular}{|c|c|c|c|c|}
\hline Side ratio(D/B) & $\mathbf{B}(\mathbf{c m})$ & $\mathbf{D}(\mathbf{c m})$ & $\mathbf{H}(\mathbf{c m})$ & weight(g) \\
\hline 2 & 7.1 & 14.2 & 80 & $1000 \mathrm{~g}$ \\
\hline
\end{tabular}


TABLE III.

TLD CASES

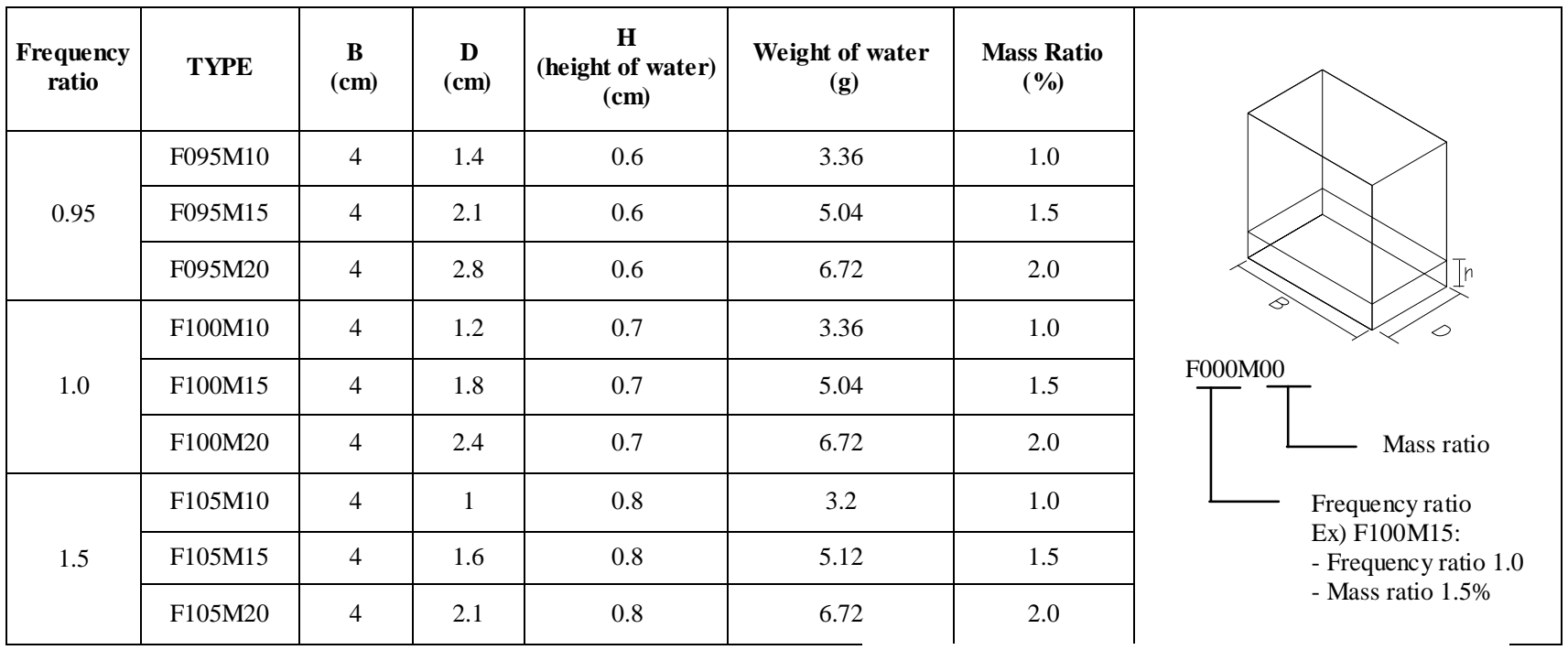

\section{DISPLACEMENT RESPONSE}

As the experimental technique, in the case of the TLD model on the aeroelastic model of side ratio 2 was not installed, displacement response in across-wind ( $Y$ direction) was measured, and then the aeroelastic experiment was conducted after installing the nine TLD models, which are classified according to mass ratio and natural frequency ratio, on the top floor of the aeroelastic model. Experimental wind speed was increased at $0.5 \mathrm{~m} / \mathrm{s}$ intervals between $0.8 \mathrm{~m} / \mathrm{s}$ and $2.5 \mathrm{~m} / \mathrm{s}$. Sampling frequency is $200 \mathrm{~Hz}$, and the data were measured 5 times, with 2048 measurements each time. Fig. 3 show vibration displacement response in across wind where the natural frequency ratio is $0.95,1.1$, and 1.05 for each mass ratio 1 , 1.5 , and 2. In case of no TLDs, the wind speed that shows maximum displacement response is the speed that causes vortex induced vibration, and the wind speed is $2.1 \mathrm{~m} / \mathrm{sec}$ when side ratio is 2.The difference in vibration displacement response when there is no TLD on the top floor of the model, and the vibration displacement response when TLD is installed on the top floor, according to the change of mass ratio was examined. The result shows that the decrease of vibration displacement response according to the change of mass ratio and frequency ratio reaches from a minimum of $30 \%$ to a maximum of $60 \%$. 1) When it comes to the change of mass ratio, the decrease of vibration displacement response is maximum at mass ratio $1.5 \%$, and as the mass ratio increases to $2 \%$, the effect of the decrease of vibration displacement response becomes smaller than in case of mass ratio $1.5 \%$. 2) When it comes to the change of frequency ratio, it is shown in the order of tuning ratio (natural frequency of aeroelastic model/natural frequency of TLD model) $1.0>0.95>1.05$, and the biggest decrease effect of vibration displacement response could be seen at tuning ratio 1 .

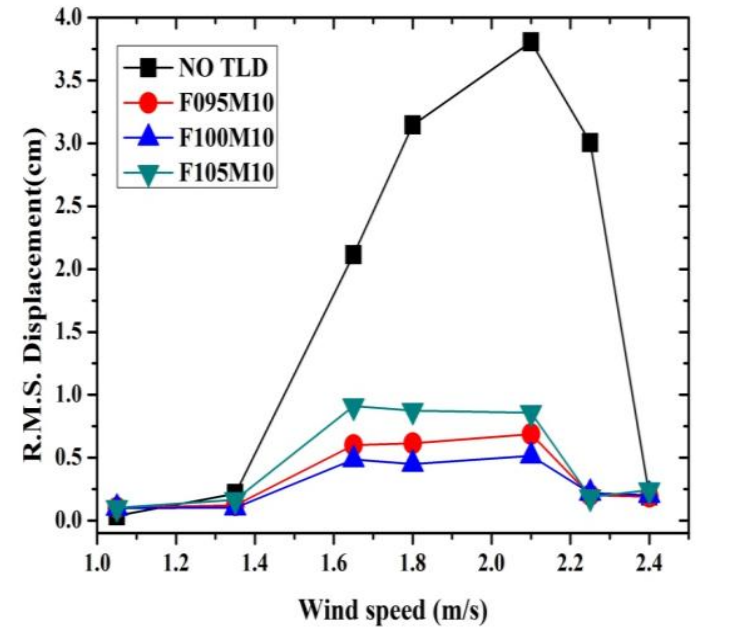

Figure 3. (a) mass ratio $1 \%$

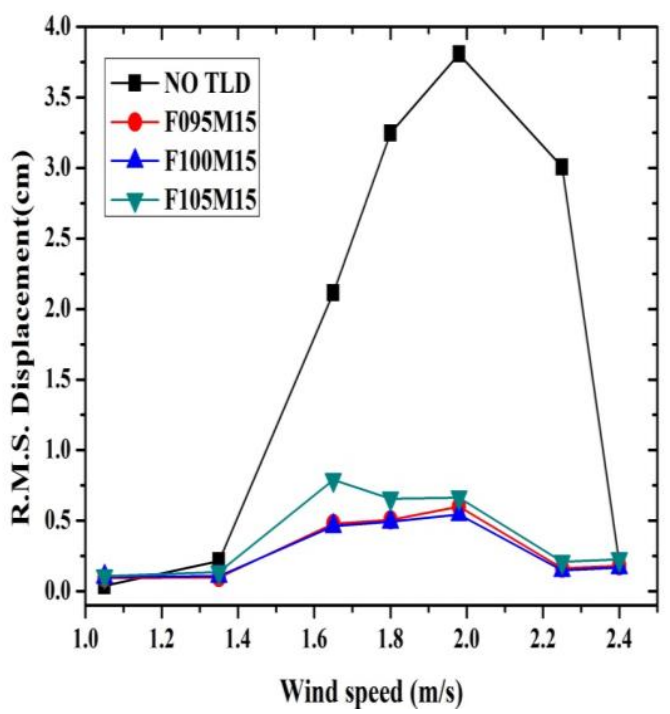

Figure 4. (b) mass ratio $1.5 \%$ 


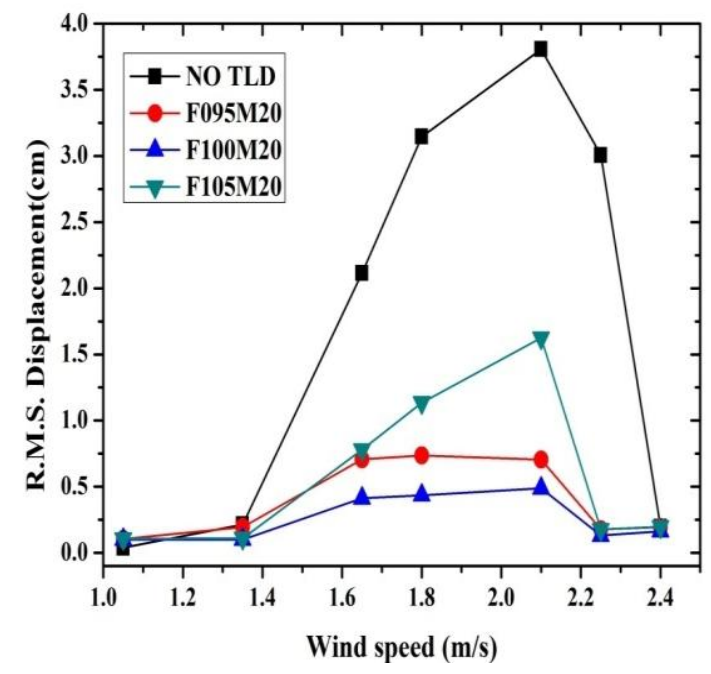

Figure 5. (c) mass ratio $2 \%$ Across-wind response of side ratio 2

\section{CONCLUSION}

As for the reduction effects in vibration displacement responses due to vortex-induced vibrations occurring in the low wind speed section of the high-rise building, the reduction was $30 \%$ at the minimum and $60 \%$ at the maximum in accordance with the mass ratios and frequency ratios of the TLD model installed on top of the high-rise building. The natural frequency of the TLD model was within the range of $0.95-1.05$ in consideration of the optimal frequency The greatest reduction effects in vibration displacement responses at the tuning ratio 1 in the order of $1.00>0.95>1.0$ with respect to tuning ratio changes in accordance with natural frequency ratios.

\section{ACKNOWLEDGMENT}

The This work is supported by the National Research Foundation of Korea (NRF) grant funded by the Korea government. (MEST) (NO.2011-0028567).

\section{REFERENCES}

[1] A. Kareem, "Reduction of wind induced motion utilizing a tuned sloshing damper", Journal of Wind Engineering and Industrial Aerodynamics, 1990, vol. 36, pp. 725-737.

[2] A. Kareem, "Mitigation of Wind Induced Motion of Tall Buildings. Journal of Wind Engineering and Industrial Aerodynamics, 1983, dvol.11, pp. 273-284.

[3] A. Kareem, T. Kijewski and Y. Tamura, "Mitigation of motion of tall buildings with recent applications", Wind and Structures. 1999, vol. 2(3), pp. 201-251.

[4] V.J. Modi and F. Welt, "Vibration control using nutation dampers", Proceeding on Flow Induced Vibration. England, 1987, pp. 369376.

[5] T. Noji, H. Yoshoda, E. Tatsumi and H. Kosaka, "Study on vibration control damper utilizing sloshing of water", Journal of Wind Engineering, 1988, vol.37, pp. 557-566.

[6] Y. Fujino, L.M. Sun, B.M.Pacheco, "Tuned liquid damper(TLD) for Suppressing horizontal motion of structures", Journal of Engineering Mechanics ASCE. 1992, vol.118(10), pp. 2017-2030. 